\title{
Receptive Fields of Second-Order Taste Neurons in Sheep
}

\author{
Convergence of Afferent Input Increases \\ During Development ${ }^{a}$
}

\author{
MARK B. VOGT AND CHARLOTTE M. MISTRETTA ${ }^{b}$ \\ Department of Oral Biology \\ School of Dentistry and \\ Center for Human Growth and Development \\ University of Michigan \\ Ann Arbor, Michigan 48109
}

Previous neurophysiological studies have provided only a gross regional characterization of the lingual receptive fields of mammalian central taste neurons. Here we report the preliminary results of a quantitative analysis of the receptive fields of secondorder taste neurons in terms of discrete receptor populations, and present evidence for an increase in afferent convergence on central taste neurons in sheep during development.

Afferent neurons in the chorda tympani nerve (CT) innervate taste buds located in fungiform papillae on the anterior tongue and synapse centrally on neurons in the nucleus of the solitary tract (NST) in the medulla. CT fibers ${ }^{1}$ or NST neurons were studied in fetal sheep aged about 130 days gestation (term $=147$ days) and lambs aged 40-50 days postnatal. Single neurons were isolated and responses to $0.5 \mathrm{M}$ $\mathrm{NH}_{4} \mathrm{Cl}, \mathrm{NaCl}$, and $\mathrm{KCl}$ were recorded. Then a fine platinum probe was used to electrically stimulate ( 10 microamps anodal current) individual fungiform papillae and the number and location of papillae in the receptive field of the neuron were recorded. The criterion for a chemical or electrical response was that impulse frequency should be greater than the mean plus two standard deviations of the spontaneous rate.

Most neurons had a receptive field located in the area of the highest concentration of fungiform papillae: the edge of the anterior one-third of the tongue extending about $1.5 \mathrm{~cm}$ on both the dorsal and ventral surfaces. In general, the fungiform papillae in the receptive field of a particular neuron were located together and were not scattered among other papillae. We also found there could be considerable overlap in the receptive fields of different neurons, that is, they might share many of the same papillae.

As presented in TABLE 1, the receptive field size of NST neurons was generally greater than that of CT neurons. Furthermore, the receptive field size of NST neurons was larger in lambs than fetuses, but for CT neurons receptive field size did not

\footnotetext{
${ }^{a}$ Supported by National Science Foundation Grant BNS 83-11497 to CMM.

${ }^{b}$ Address for correspondence: Charlotte M. Mistretta, Department of Oral Biology, School of Dentistry, University of Michigan, Ann Arbor, MI 48109.
} 
TABLE 1. Receptive Field Size of CT and NST Neurons in Fetus and Lamb

\begin{tabular}{lccc}
\hline & & \multicolumn{2}{c}{$\begin{array}{c}\text { Number of Papillae } \\
\text { in Receptive Field }\end{array}$} \\
\cline { 3 - 4 } $\begin{array}{c}\text { Neuron } \\
\text { Type }\end{array}$ & $\begin{array}{c}\text { Format of } \\
\text { Results }\end{array}$ & Fetus & Lamb \\
NST neurons & $\bar{X}^{a}$ & 21 & 37 \\
& $n(\mathrm{SD})$ & $8(10)$ & $30(20)$ \\
range & $8-36$ & $2-100$ \\
CT fibers & $\overline{\mathrm{X}}$ & 13 & 11 \\
& $n(\mathrm{SD})$ & $17(9)$ & $32(7)$ \\
& range & $2-40$ & $2-29$ \\
\hline
\end{tabular}

${ }^{a} \overline{\mathrm{X}}=$ mean, $n=$ number of fields, $\mathrm{SD}=$ standard deviation, range $=$ smallest-largest receptive field.

increase (Age [fetus, lamb] by neurons [CT, NST] ANOVA; neurons and interaction Fs $[1,83]>6.0, p s<0.02$ ).

Although these data are preliminary, the larger receptive fields of NST neurons compared to peripheral neurons indicates a convergence of taste papilla input on central neurons. Furthermore, because the average receptive field size of NST neurons is larger in lambs than fetuses, the degree of convergence increases during development. Apparently this is due to increasing convergence of CT fibers onto NST neurons, since the total number of papillae innervated by a CT fiber does not increase between fetal and lamb ages.

\section{REFERENCE}

1. Mistretta, C. M., T. Nagai \& R. M. Bradley. Relation of receptive field size and salt taste responses in chorda tympani fibers during development. Ann. N.Y. Acad. Sci. This volume. 